\title{
A New Algorithm to Reduce Peak to Average Power Ratio in OFDM Systems based on BCH Codes
}

\author{
Brahim BAKKAS $^{1}$, Reda Benkhouya ${ }^{2}$, Toufik Chaayra ${ }^{3}$, Chana Idriss $^{4}$, Hussain Ben-Azza ${ }^{5}$ \\ Ecole Nationale d'Art et Méties (ENSAM-Meknès), Moulay Ismail University, Meknès 50050, Morocco ${ }^{1,5}$ \\ MISC-Faculty of Sciences, Ibn Tofail University, Kenitra 14000, Morocco ${ }^{2}$ \\ Department of Mathematics and Computer, Multidisciplinary Faculty of Nador, Mohammed I University, Morocco ${ }^{3}$ \\ High School of Technology, Moulay Ismail University, Meknès 50050, Morocco ${ }^{4}$
}

\begin{abstract}
Orthogonal Frequency Division Multiplexing (OFDM) has a great peak-to-average power ratio (PAPR). This will reduce the performance of the power amplifier (PA). Therefore, PAPR deteriorates the overall energy efficiency of an OFDM system. Peak Insertion (PI) is one of the most commonly used methods to reduce PAPR, it gives the best reduction in PAPR. Therefore, it causes a strong degradation in Bit Error Rate (BER). To solve this problem, we propose a new algorithm called BCB-OFDM based on Bose Chaudhuri Hocquenghem Codes (BCHs) and PI. BCB is implemented in OFDM system with Quadrature Amplitude Modulation (QAM) and two coding rates $1 / 2$ and $1 / 4$ over an Additive White Gaussian Noise (AWGN) channel. Simulation results show that the BCB is very interesting and achieve a good value in terms of PAPR reduction with keeping good performance compared with PI and normal OFDM. In addition, BCB algorithm is simple, robust, and leaves no requirement side information with more flexibility to choose between PAPR reduction and BER performances.
\end{abstract}

Keywords-Orthogonal Frequency Division Multiplexing (OFDM); Peak to Average Power Ratio (PAPR); Bit Error Rate (BER); Peak Insertion (PI); Coding; Bose Chaudhuri Hocquenghem (BCH)

\section{INTRODUCTION}

Orthogonal Frequency Division Multiplex (OFDM) is a multi-carrier technique that has shown its effectiveness, robust against interference problems caused by multipath. Thanks to its simplicity of implementation through the use of Fourier Transform. OFDM is the basic technology used in wireless communication such as WiFi, WiMAX [1], [2], [3], 4G [4] and 5G [5], [6], [7], [8]. However, it has a major drawback caused by the high value of Peak To Average Power Ratio (PAPR) defined as the ratio between the maximum power and the average power of an OFDM signal. The high value of PAPR value forces the Power Amplifier (PA) to work in the non-linear region and cause a degradation of the signal and need a large consumption in energy [9], [10].

Several approaches to resolving this problem have been offered, the most widely utilized approaches are: Selective Mapping Technique (SLM)[11], Partial Transmit Sequences (PTS) [12], [13], companding [14], [3], Clipping [15], Palm Clipping [16], Peak Insertion (PI)[17] and Tone reservation [18]. In [19], [20], the authors compare some PAPR reduction methods in terms of PAPR and BER. The obtained result in this comparison is that the Peak Insertion technique proposed gives the highest PAPR reduction, but it causes a high fort degradation [21]. Also, linear codes give the best result in terms of BER with small PAPR reduction by finding the best code with a diminished PAPR however it produces a computational complexity to find the best code word with a low PAPR value [22], [23], [24]. As a result, to choosing the relevant PAPR reduction approach, a compromise between PAPR reduction and BER performances must be made.

The main contributions of this article are: (1) Proposed a new algorithm to reduce PAPR in OFDM Systems called BCB algorithm based on BCH codes and Peak Insertion. (2) Using $\mathrm{BCH}$ codes to explore the Peak Insertion to improve its signal degradation. (3) $\mathrm{BCB}$ method does not require any site information from the transmitter.

The remainder of this paper is organized as follows: The System model and PAPR definition and related work about Peak Insertion are presented in Section II] The proposed method and algorithm, its principle and parameters are detailed in Section III] In Section IV] we provide and discuss the simulation results in terms of PAPR reduction and BER performance compared with the normal OFDM and PI method. The conclusion and future works are presented in Section $\mathrm{V}$

\section{OFDM SYSTEM AND RELATED WORK}

In this section, we present the OFDM system model and related work about peak insertion method.

\section{A. OFDM System Model and PAPR Definition}

The principle of the OFDM system is composed by two parts as presented in Fig. 1. At the transmitter side, the input data are mapped by one of the modulation schemes. The obtained signal is modeled by using the Inverse Fast Fourier Transformation (IFFT) algorithm with N-points before being transferred to the channel. At the receiver side, the process inverse is performed. The discrete OFDM signal in the timedomain is defined by:

$$
x(n)=\frac{1}{\sqrt{N}} \sum_{k=0}^{N-1} X_{k} * e^{\left(\frac{j 2 \pi n k}{N}\right)}, \quad 0 \leq n \leq N-1 .
$$

Where $N$ is the number of subcarriers, and $X_{k}$ is the $k$ th subcarrier of the same OFDM symbol. The PAPR is defined as the ratio of maximal power and the average power :

$$
P A P R=\frac{\max \left(|x(n)|^{2}\right)}{E\left\{|x(n)|^{2}\right\}}, \quad 0 \leq n \leq N-1,
$$




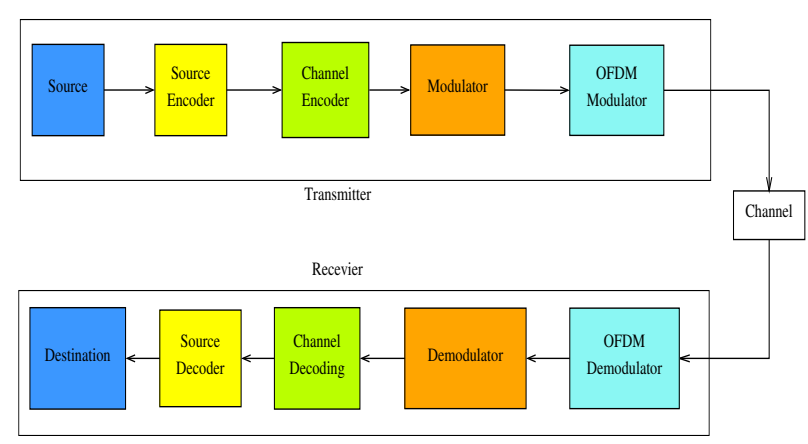

Fig. 1. Communication Digital Systems Block with OFDM.

where $E\{$.$\} represents the expectation value. PAPR perfor-$ mance is represented by the Complementary Cumulative Distribution Function (CCDF), which is the probability of PAPR exceeding a threshold, defined as follows:

$$
C C D F(\gamma)=\operatorname{Pr}[P A P R \geq \gamma],
$$

where $\gamma$ is a threshold value.

\section{B. Peak Insertion Method}

Peak Insertion (PI) method proposed in [17] is implemented by adding an impulse $\alpha$ to the original OFDM symbol in the frequency domain to reduce the PAPR value. The resulting signal $Y_{k}$ of a signal $X_{k}$ is given by:

$$
Y_{k}=X_{k}+\delta\left(k-k_{\alpha}\right)
$$

where $X_{k}$ is the original OFDM symbol, $\alpha$ is a positive real number representing the strength of the inserted peak, $k_{\alpha}$ is the position of the inserted peak, $N$ is the number of subcarriers [17]. The peak $\alpha$ is inserted to increase the PAPR of the signal in the frequency domain. However, after PI the mean square value of this signal will increase, but with a greater mean square value [19]. The power of this signal is then controlled by scaling it by a real $\beta$ such that its mean square value is reduced to a suitable level without affecting the PAPR reduction [17]. At the receiver, To restore the original OFDM, reverse operations are carried out. PI method has a benefit in PAPR reduction with a gain of $8 \mathrm{~dB}$ [23], [17]. However, it presents the drawback of signal degradation, in this area we present a new algorithm to reduce PAPR with good performances in terms of BER .

\section{Proposed Method BCB PAPR}

In this Section, we presents the principle of the proposed method based on $\mathrm{BCH}$ codes and PI technique. The BCH codes form a large class of error correcting cyclic codes, This class of codes is used for purpose of multiple-error corrections. For any positive integers $m$ with $m \geq 3$, and, $t$ with $t<2^{m-1}$, there exists a binary $\mathrm{BCH}$ code with the following parameters:

$$
\begin{array}{ll}
n & : \text { Block length } n=2^{m}-1 \\
k & : \text { BCH Code length } k=2^{m}-m-1 \\
(n-k) & : \text { Number of parity-check digits }(n-k) \geq m t \\
d & : \text { Minimum distance } d \geq 2 t+1
\end{array}
$$

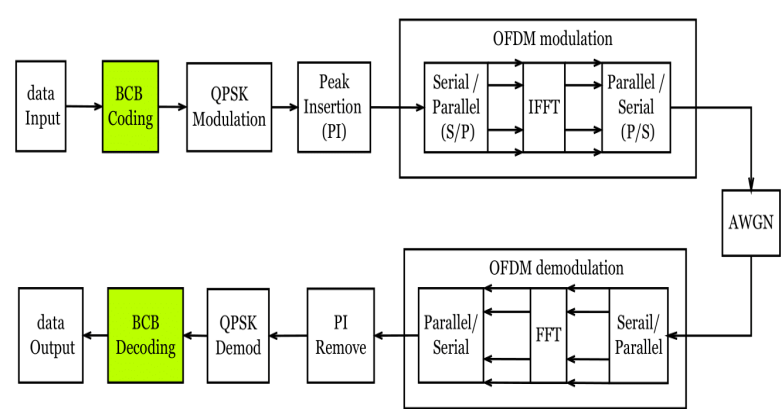

Fig. 2. BCB Peak Insertion in OFDM Systems.

We call this code a $t$-error-correcting $\mathrm{BCH}$ code, and we note in this paper the $B C H(n, k)$ a $\mathrm{BCH}$ code.

To improve the Peak Insertion technique, we propose a new method combined with $\mathrm{BCH}$ codes noted in this paper BCB-OFDM reduction. Let $B C B(n, k, v, \alpha)$ be the new block coding scheme with peak insertion $\alpha$ where:

$$
\begin{array}{ll}
n, k & : \text { Block length and code length respectively } \\
v & : \text { Number of bits information } \\
\alpha & : \text { Peak insertion value } \\
(k-v) & : \text { length of zeros padding }
\end{array}
$$

Let $m^{(i)}=b_{1}^{(i)}, b_{2}^{(i)}, \ldots, b_{v}^{(i)}$ is the $i^{\text {th }}$ block bits of size $v$, where $i=1,2 \ldots$, nbits, and nbits $=\log _{2}(M)$ number of bits/symbols. The $m^{(i)}$ message with length $v$ bits is padded with $(k-v)$ to have a block with $k$ bits noted by $B^{(i)}$ where $k$ is the $\mathrm{BCH}$ code length. The block $B^{(i)}$ is now represented as bellow:

$$
B^{(i)}=b_{1}^{(i)}, \ldots, b_{v}^{(i)}, \overbrace{0, \ldots, 0}^{(k-v)}
$$

We apply the $\operatorname{BCH}(n, k)$ encoding, the encoded message $C^{(i)}$ of $B^{(i)}$ is given as fellow:

$$
C^{(i)}=b_{1}^{(i)}, \ldots, b_{v}^{(i)}, \overbrace{0, \ldots, 0}^{(k-v)}, c_{n-k+1}^{(i)}, \ldots, c_{n}^{(i)}
$$

With 4-QAM modulation we have 2 bits for each symbol, then the coded message $C$ is now construct respectively from two blocks $C^{(1)}$ and $C^{(2)}$ by flipping the zeros block of each $C^{(i)}$ to the middle of the codded message $C$ as follows:

$$
\begin{array}{r}
C=b_{1}^{(1)}, \ldots, b_{v}^{(1)}, c_{n-k+1}^{(1)}, \ldots, c_{n}^{(1)}, \overbrace{0, \ldots, 0}^{2(k-v)+1}, \\
b_{1}^{(2)}, \ldots, b_{v}^{(2)}, c_{n-k+1}^{(2)}, \ldots, c_{n}^{(2)}
\end{array}
$$

The coded message $C$ is now mapped by 4QAM modulation, $X=\left[X_{0}, X_{1}, \ldots, X_{N-1}\right]$, a peak real $\alpha$ is inserted before using the $\mathrm{N}$-points IFFT algorithm to obtained the OFDM message $x=\operatorname{IFFT}\left(X^{T}\right)$ where $(.)^{T}$ is the complex transpose. Then the signal OFDM is passed through an AWGN channel. At the received part, the inverses process is applied. Perform Serial to Parallel conversion, then the $N$-point FFT is applied. The peak $\alpha$ is removed from the signal before applying 4QAM demodulation. The decoding algorithm is applied and the bits informations is recovered and compared 
with the original bits to compute the BER for each SNR as shown in Fig. 2. The main steps of the proposed method have been widely described in the following Algorithm 1 .

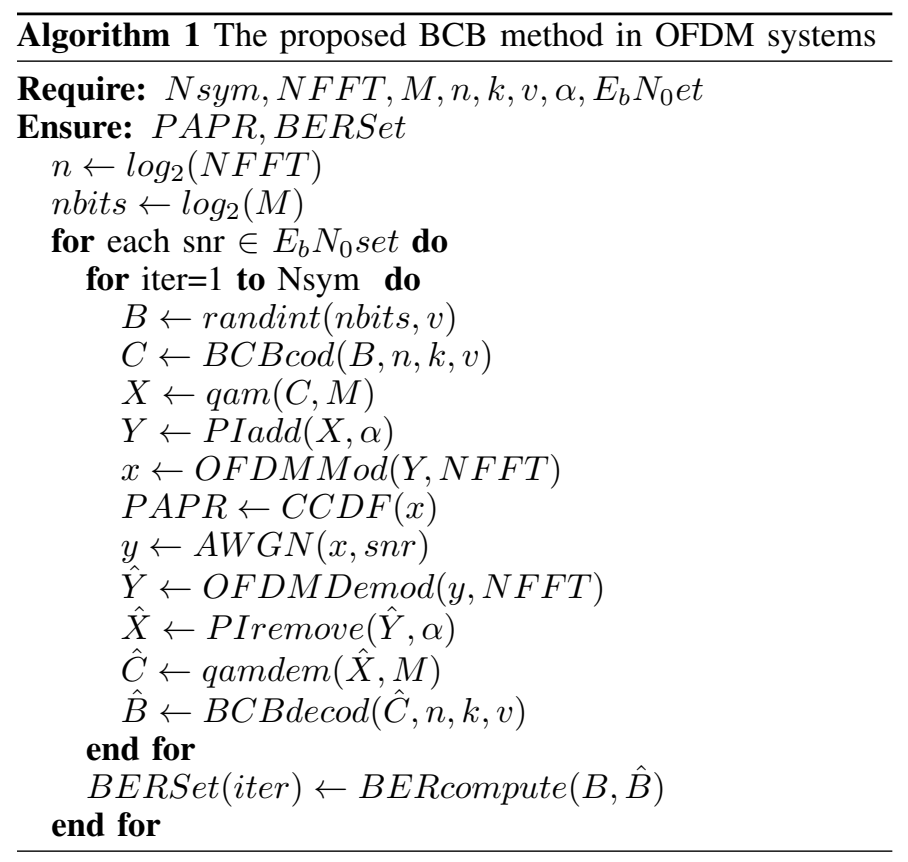

\section{Simulation Results And Discussion}

The performance of the proposed algorithm is investigated in terms of PAPR reduction and BER performances. We start this section by examining the impact of $\alpha$ and coding rates (1/4 and 1/2) respectively. The results are represented for each case. Finally, we compare the proposed algorithm with the normal OFDM and the Peak Insertion. The simulations are carried out with 4QAM (QPSK) as modulation schemes with N-points NFFT size equal to 256 over an AWGN channel. A number $N$ sym of OFDM symbols are generate randomly, we note that in this system we have neglected the other parameters of the OFDM systems such as cyclic prefix and guard interval. Table I detailed the parameters of simulation, and Table II] regroups the possible value of $\mathrm{BCH}$ coding, we choose two values $k$ equal to 207 and 147 (respectively with low and high capacity errors correcting).

TABle I. Simulation Parameters of the Proposed Algorithm BCB IN OFDM SYSTEMS

\begin{tabular}{cc}
\hline Parameter & values \\
\hline Number of symbols (Nsym) & 10000 \\
Number of bit errors (Nberr) & 100 \\
FFT size (NFFT) & 256 \\
Peak insertion $\alpha$ & $0,29,50$ and 80 \\
Peak insertion $\beta$ & 1 \\
BCH $(\mathrm{n}, \mathrm{k}, \mathrm{t})$ & $\mathrm{n}=255, \mathrm{k}=207$ and 147 (see Table II \\
Message Rate $(\mathrm{h})$ & $1 / 2$ and $1 / 4$ \\
Modulation scheme & 4-QAM (QPSK) \\
Channel model & AWGN \\
\hline
\end{tabular}

\section{A. PAPR Performances of the Proposed BCB}

In this subsection, we fix some parameters such as FFT size $N F F T=256$, information size (message) $v=64$ and $v=128$. we choose two value of coding $k$ equal to 147 and
TABLE II. Possible VALues of $B C H(n=255, k, t)$ CODES

\begin{tabular}{cc|cc|cc|cc}
\hline $\mathrm{k}$ & $\mathrm{t}$ & $\mathrm{k}$ & $\mathrm{t}$ & $\mathrm{k}$ & $\mathrm{t}$ & $\mathrm{k}$ & $\mathrm{t}$ \\
\hline 247 & 1 & 187 & 9 & 123 & 19 & 63 & 30 \\
239 & 2 & 179 & 10 & 115 & 21 & 55 & 31 \\
231 & 3 & 171 & 11 & 107 & 22 & 47 & 42 \\
223 & 4 & 163 & 12 & 99 & 23 & 45 & 43 \\
215 & 5 & 155 & 13 & 91 & 25 & 37 & 45 \\
$\mathbf{2 0 7}$ & $\mathbf{6}$ & $\mathbf{1 4 7}$ & $\mathbf{1 4}$ & 87 & 26 & 29 & 47 \\
199 & 7 & 139 & 15 & 79 & 27 & 21 & 55 \\
191 & 8 & 131 & 18 & 71 & 29 & 13 & 59 \\
\hline
\end{tabular}

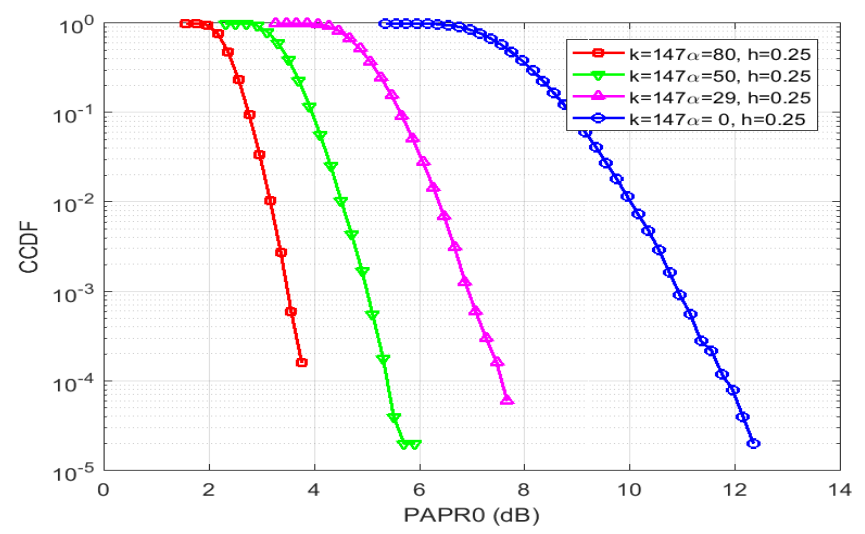

Fig. 3. CCDF Curves of the Proposed BCB-OFDM NFFT=256, 4QAM, $B C B(n=255, k=147, v=64, \alpha)$ with Variable $\alpha$.

207. Also, we varying the value of the peak $\alpha$ to $0,29,50$ and 80 as mentioned in Table I. The results are depicted by using the CCDF curves for each cases. Firstly, we set the rate to $1 / 4$, the results are presented with the CCDF curves with variable $\alpha$ in Fig. 3 and Fig. 4. Secondly we change the rate to $1 / 2$ the results are showed in Fig. 5 and Fig. 6

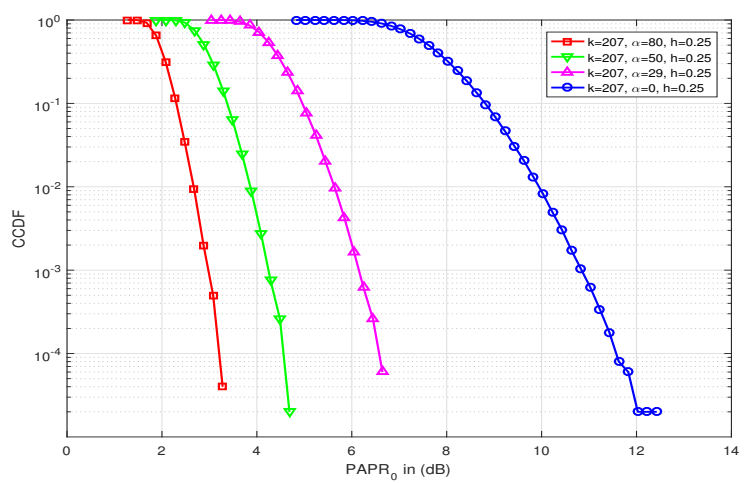

Fig. 4. CCDF Curves of the Proposed BCB-OFDM NFFT=256, 4QAM, $B C B(n=255, k=207, v=64, \alpha)$ with Variable $\alpha$.

In the first case, $B C B(n=255, k=147, v=64, \alpha)$, we set $k$ to $147, v$ to 64 and the number of zero padding is $2 *(k-v)$ with variable $\alpha$. The result are depicted in terms of PAPR in Fig. 3 From the obtained results a significant reduction in terms of PAPR is observed for $\alpha$ is equal to 29 where the $8 \mathrm{~dB}$ is attend. Also, we achieves $6 \mathrm{~dB}$ when $\alpha$ is great then 29.

Fig. 4 shows the CCDF with variable $\alpha$ of the second case 


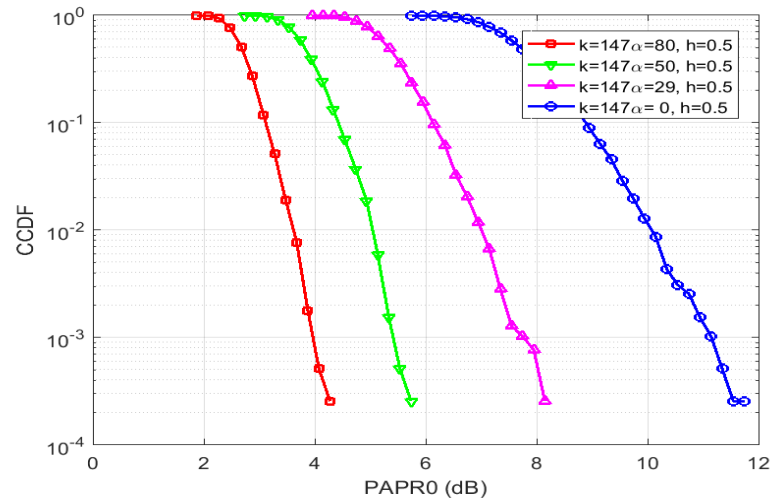

Fig. 5. CCDF Curves of the Proposed BCB-OFDM NFFT=256, 4QAM, $B C B(n=255, k=147, v=128, \alpha)$ with Variable $\alpha$.

$B C B(n=255, k=207, v=64, \alpha)$. In this case we set $k$ to 207 and we keep other parameters $N F F T=256, v=64$. From this figure we note that proposed method achieve a good reduction even we augment the peak $\alpha$. The value of PAPR is reduce to half (more then $6 \mathrm{~dB}$ ) when $\alpha$ is great then 50 , the gain is very important and it is between $7 d B$ and $9 d B$.

Now, we repeat the same simulation realized in the first and second cases by changing the message size from $v=64$ to $v=128$ with a rate equal to $1 / 2$ and two values of $\mathrm{BCH}$ codes length $k$.

In the third case, $B C B(n=255, k=147, v=128, \alpha)$ the value coding length $k$ is set to 147 and the number of zero padding is $2^{*}(\mathrm{k}-\mathrm{v})$ with variable $\alpha$. The result are depicted in Fig. 5 in terms of PAPR with variable $\alpha$. As shown in this figure, the, one can clearly see that the PAPR decreases when $\alpha$ increases, a value of $4.2 \mathrm{~dB}$ achieved at $\mathrm{CCFD}=10^{-4}$ when $\alpha=80$.

In the forth case, $B C B(n=255, k=207, v=128, \alpha)$ we set the coding length $k$ to 207 and the number of zero padding is $2 *(k-v)$. with variable $\alpha$. The CCDF curves of the proposed algorithm is showed in Fig. 6 In This figure, we notice that the PAPR values reduced when the $\alpha$ increases. The value $3.8 \mathrm{~dB}$ is achieved at $\mathrm{CCDF}=10^{-} 4$ when $\alpha$ equal to 80 .

\section{B. Comparative Studies}

To validate the proposed method in terms of PAPR and BER performances, we compare the BCB method with Peak Insertion and normal OFDM by varying the code rate and value of the peak $\alpha$. Firstly, we depict the CCDF and BER curves with variable $\alpha$, coding rate, and information size, the results are depicted in Fig. 7 for each value of $\alpha$. Finally, the gain in terms of PAPR at $\mathrm{CCDF}=10^{-4}$ versus variable $\alpha$ are summarized in Table III while Table IV regroups the SNR loss in $\mathrm{dB}$ calculated in eq. (8) with variable $\alpha$, coding length $k$ and information size $v$.

$$
S N R_{\text {loss }}=S N R_{\text {method }}-S N R_{\text {Normal }}
$$

According to the previous results depicted in Fig. 7, and summarize results in Tables [IV and [II] The value of PAPR
TABLE III. PAPR GAIN AT CCDF $=10^{-4}$ of the Proposed Method, PEAK INSERTION AND NORMAL OFDM

\begin{tabular}{ccccc}
\hline Method & $\alpha=0$ & $\alpha=29$ & $\alpha=50$ & $\alpha=80$ \\
\hline OFDM & 0 & 6.4 & 8.2 & 9.3 \\
PI $(\alpha)$ & 0 & 4.2 & 6.2 & 7.9 \\
BCB $(255,147,64, \alpha)$ & 0 & 5.4 & 6.6 & 8.8 \\
BCB $(255,207,64, \alpha)$ & 0 & 3.8 & 6.0 & 7.6 \\
BCB $(255,147,128, \alpha)$ & 0 & 4.2 & 6.2 & 7.9 \\
BCB $(255,207,128, \alpha)$ & 0 & &
\end{tabular}

diminishes where $\alpha$ increases. It is worth remarking here that the best gain in terms of PAPR is achieved when the value of $\alpha$ is great than 50. The introduction of block coding let us correct some errors with the same PAPR as the normal OFDM. Overall we see the technique proposed here easily outperforms the other approaches in terms of BER with good values in terms of PAPR.

TABLE IV. SNR Loss in $d B$ of The Proposed Method, PEAK INSERTION AND NORMAL OFDM AT BER $=10^{-4}$

\begin{tabular}{ccccc}
\hline Method & $\alpha=0$ & $\alpha=29$ & $\alpha=50$ & $\alpha=80$ \\
\hline OFDM & & \multicolumn{3}{c}{0} \\
PI $(\alpha)$ & 0 & 6.4 & 10.4 & 14.4 \\
BCB $(255,147,64, \alpha)$ & -4.5 & 0.3 & 3.4 & 7.5 \\
BCB $(255,207,64, \alpha)$ & -3.0 & 1.0 & 5.5 & 9.5 \\
BCB $(255,147,128, \alpha)$ & -5.7 & 0.5 & 4.5 & 8.5 \\
BCB $(255,207,128, \alpha)$ & -5.5 & 2.0 & 6.0 & 10.0 \\
\hline
\end{tabular}

\section{CONCLUSiON}

In this paper, we have proposed a new method to reduce PAPR and BER in the OFDM system based on BCH codes and Peak Insertion. The proposed algorithm achieved a good reduction in terms of PAPR reduction with good performances in terms of BER compared with Peak Insertion. Varying the parameter $\alpha$ we achieve a good reduction with a gain of $8.8 \mathrm{~dB}$ when $\alpha$ is equal to 80 with a gain of $6 \mathrm{~dB}$ in terms of BER compared with PI (with $\alpha=80$ ). The simulation results show that the proposed method is simple, robust, and does not need side information with more flexibility to choose between PAPR reduction and BER performances. Future work is planned to study the proposed algorithm more in-depth by using Genetic

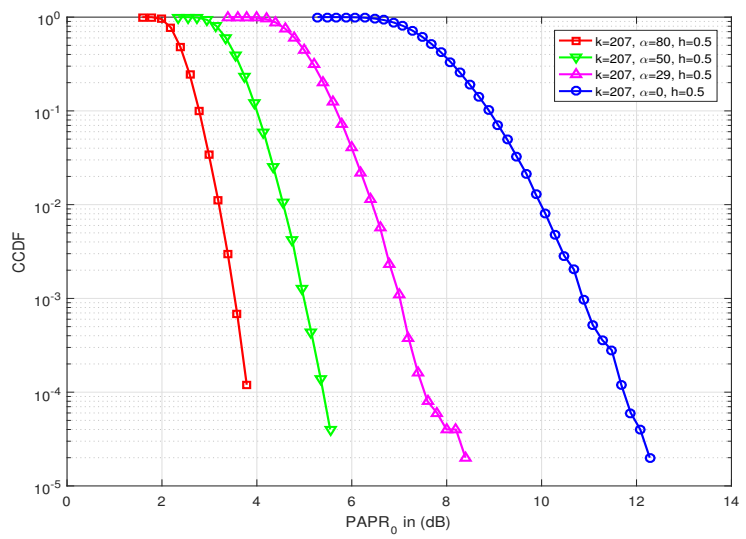

Fig. 6. CCDF Curves of the Proposed BCB-OFDM FFT=256, 4QAM, $B C B(n=255, k=207, v=128, \alpha)$ with Variable $\alpha$. 
Algorithms to enhance the proposed algorithm to find the best comprise between PAPR reduction and BER performance. Also, we investigate other coding schemes especially the Goppa codes.

\section{REFERENCES}

[1] H. Bolcskei, "Mimo-ofdm wireless systems: basics, perspectives, and challenges," IEEE wireless communications, vol. 13, no. 4, pp. 31-37, 2006.

[2] K. Fazel and S. Kaiser, Multi-carrier and spread spectrum systems: from OFDM and MC-CDMA to LTE and WiMAX. John Wiley \& Sons, 2008

[3] M. N. Aarab and O. Chakkor, "Mimo-ofdm for wireless systems: An overview," in International Conference on Artificial Intelligence and Symbolic Computation. Springer, 2019, pp. 185-196.

[4] A. Svensson, A. Ahlén, A. Brunstrom, T. Ottosson, and M. Sternad, "An ofdm based system proposal for $4 \mathrm{~g}$ downlinks," in Multi-Carrier Spread-Spectrum. Springer, 2004, pp. 15-22.

[5] B. Farhang-Boroujeny and H. Moradi, "Ofdm inspired waveforms for 5g," IEEE Communications Surveys \& Tutorials, vol. 18, no. 4, pp. 2474-2492, 2016.

[6] N. K.P and C. K. N, "Studying applicability feasibility of ofdm in upcoming 5g network," International Journal of Advanced Computer Science and Applications, vol. 8, no. 1, 2017. [Online]. Available: http://dx.doi.org/10.14569/IJACSA.2017.080128

[7] F. Sandoval, G. Poitau, and F. Gagnon, "Hybrid peak-to-average power ratio reduction techniques: Review and performance comparison," IEEE Access, vol. 5, pp. 27 145-27 161, 2017.

[8] R. Ahmed, F. Schaich, and T. Wild, "OFDM enhancements for 5G based on filtering and windowing," in Mult. Access Tech. 5G Wirel. Networks Beyond. Springer, 2018, pp. 39-61.

[9] C. Rapp, "Effects of hpa-nonlinearity on a 4-dpsk/ofdm-signal for a digital sound broadcasting signal," ESASP, vol. 332, pp. 179-184, 1991.

[10] P. Banelli, G. Baruffa, and S. Cacopardi, "Effects of hpa nonlinearity on frequency multiplexed ofdm signals," IEEE Transactions on Broadcasting, vol. 47, no. 2, pp. 123-136, 2001.

[11] S. Gupta and A. Goel, "Improved selected mapping technique for reduction of papr in ofdm systems," International Journal of Advanced Computer Science and Applications, vol. 11, no. 10, 2020. [Online]. Available: http://dx.doi.org/10.14569/IJACSA.2020.0111016

[12] Y. A. Al-Jawhar, N. S. M. Shah, M. A. Taher, M. S. Ahmed, and K. N. Ramli, "An enhanced partial transmit sequence segmentation schemes to reduce the papr in ofdm systems," International Journal of Advanced Computer Science and Applications, vol. 7, no. 12, 2016. [Online]. Available: http://dx.doi.org/10.14569/IJACSA.2016.071209
[13] Y. A. Al-Jawhar, K. N. Ramli, A. Mustapha, S. A. Mostafa, N. S. Mohd Shah, and M. A. Taher, "Reducing PAPR with Low Complexity for 4G and 5G Waveform Designs," IEEE Access, vol. 7, pp. 97 673-97688, 2019.

[14] B. Bakkas, I. Chana, and H. Ben-Azza, "PAPR reduction in MIMO-OFDM based on polar codes and companding technique," in Proc. - 2019 Int. Conf. Adv. Commun. Technol. Networking, CommNet 2019. IEEE, apr 2019, pp. 1-6. [Online]. Available: https://ieeexplore.ieee.org/document/8742379/

[15] K. Anoh, C. Tanriover, and B. Adebisi, "On the Optimization of Iterative Clipping and Filtering for PAPR Reduction in OFDM Systems," IEEE Access, vol. 5, pp. 12004-12013, 2017. [Online]. Available: http://ieeexplore.ieee.org/document/7938298/

[16] B. Bakkas, R. Benkhouya, I. Chana, and H. Ben-Azza, "Palm date leaf clipping: A new method to reduce papr in ofdm systems," Information, vol. 11, no. 4, p. 190, apr 2020. [Online]. Available: https://www.mdpi.com/2078-2489/11/4/190

[17] A. I. Siddiq, "PAPR reduction in OFDM systems using peak insertion," AEU - Int. J. Electron. Commun., vol. 69, no. 2, pp. 573-578, feb 2015. [Online]. Available: http://dx.doi.org/10.1016/j.aeue.2014.11.009

[18] B. Wang, Q. Si, and M. Jin, "A novel tone reservation scheme based on deep learning for papr reduction in ofdm systems," IEEE Communications Letters, vol. 24, no. 6, pp. 1271-1274, 2020.

[19] P. P. Ann and R. Jose, "Comparison of papr reduction techniques in ofdm systems," in 2016 International Conference on Communication and Electronics Systems (ICCES). IEEE, 2016, pp. 1-5.

[20] W. G. Abera, "Comparative study of the performances of peak-toaverage power ratio (papr) reduction techniques for orthogonal frequency division multiplexing (ofdm) signals," in International Conference on Information and Communication Technology for Develoment for Africa. Springer, 2017, pp. 56-67.

[21] P. Preenu Ann and R. Jose, "Comparison of PAPR reduction techniques in OFDM systems," Proc. Int. Conf. Commun. Electron. Syst. ICCES 2016, vol. 1, no. 4, pp. 40-49, 2016.

[22] N. Sabna, R. Revathy, and P. S. Pillai, "Bch coded ofdm for undersea acoustic links," in 2015 International Symposium on Ocean Electronics (SYMPOL). IEEE, 2015, pp. 1-6.

[23] P. Gupta, B. A. Kumar, and S. K. Jain, "Peak to average power ratio reduction in ofdm using higher order partitioned pts sequence and bose chaudhuri hocquenghem codes," in 2015 International Conference on Signal Processing and Communication Engineering Systems. IEEE, 2015, pp. 443-447.

[24] P. Kumar, A. K. Ahuja, and R. Chakka, "Bch/hamming/cyclic coding techniques: comparison of papr-reduction performance in ofdm systems," in International Conference on Intelligent Computing and Applications. Springer, 2018, pp. 557-566. 


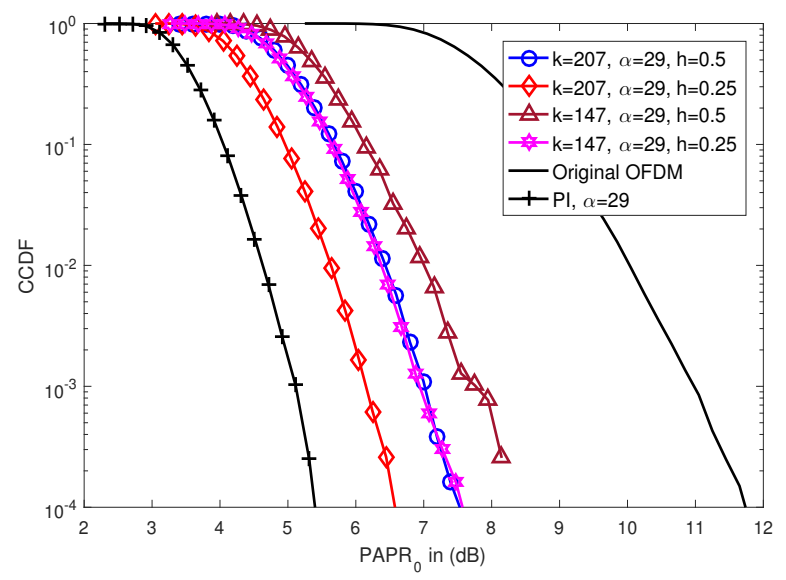

(a) $\operatorname{CCDF} \alpha=29$

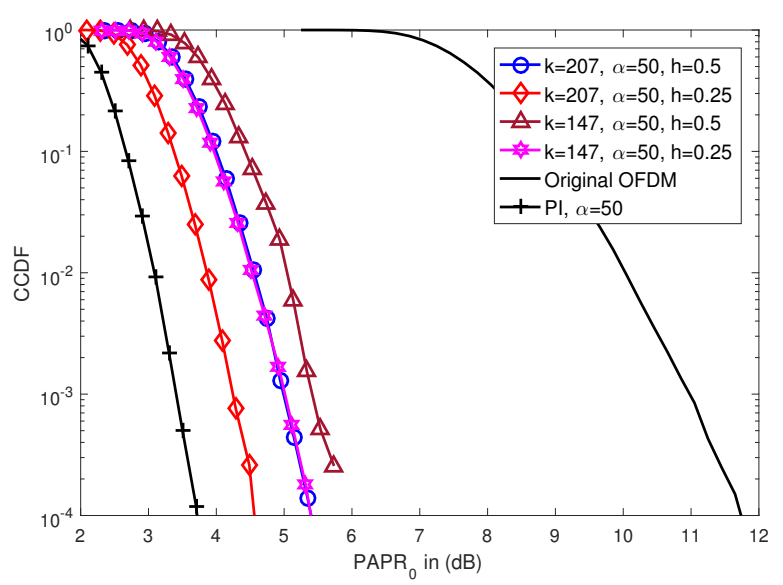

(c) CCDF, $\alpha=50$

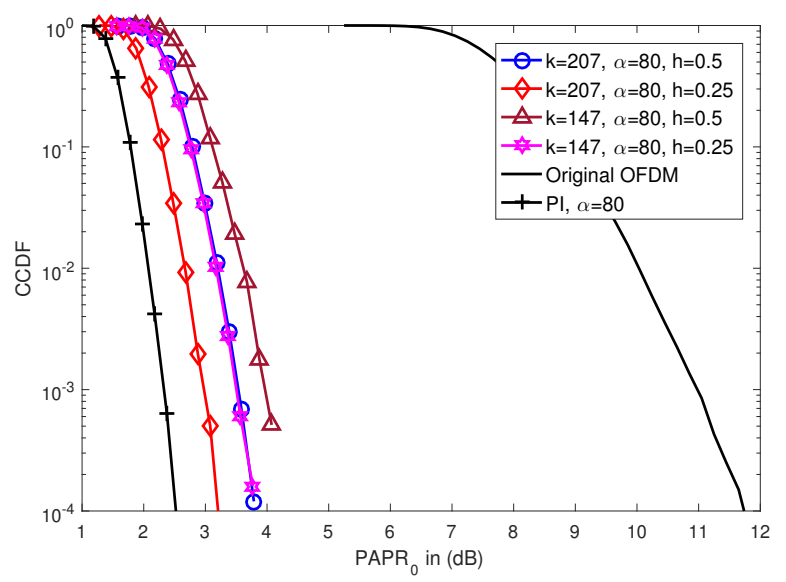

(e) CCDF, $\alpha=80$

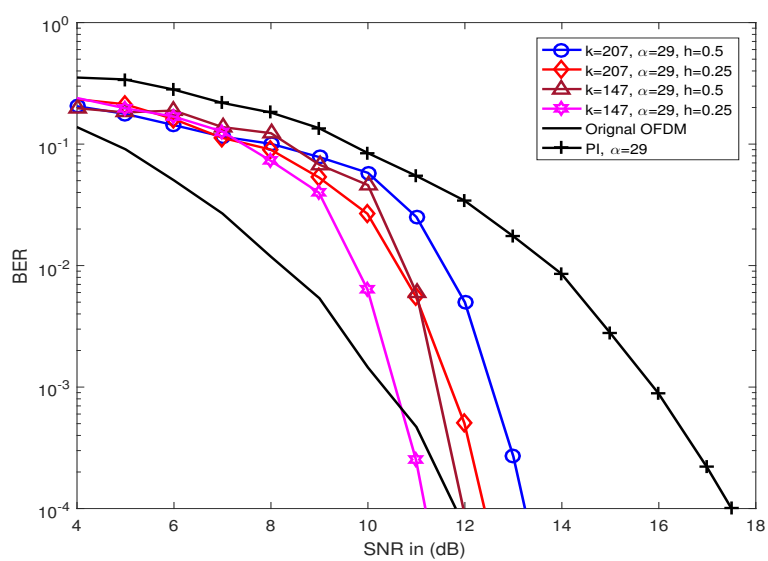

(b) BER, $\alpha=29$

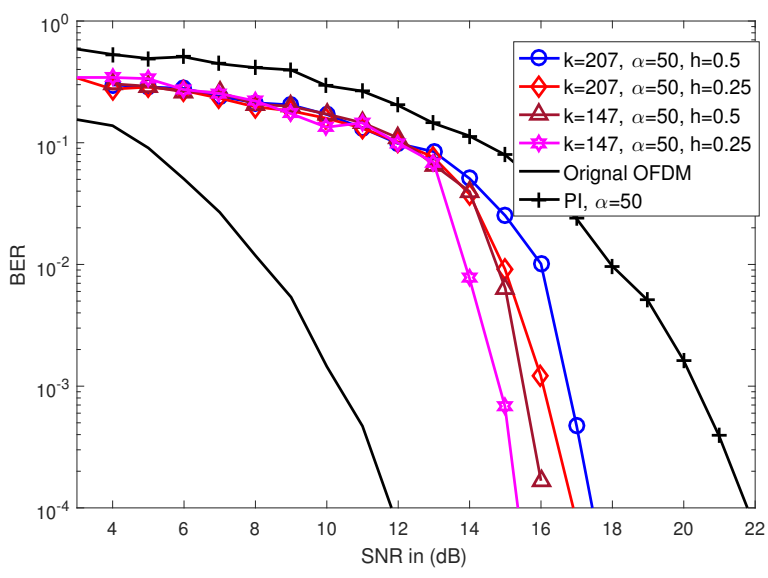

(d) BER, $\alpha=50$

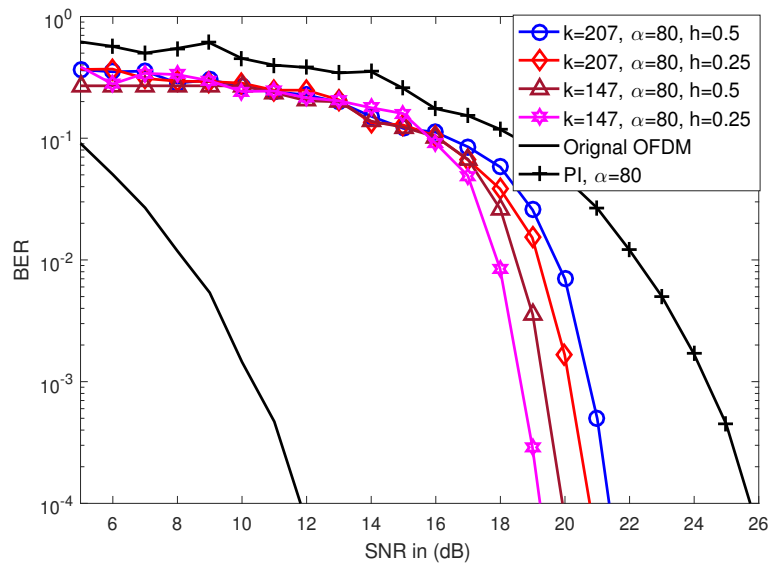

(f) BER, $\alpha=80$

Fig. 7. CCDF and BER Performances of the proposed BCB-OFDM Compared with Peak Insertion and Normal OFDM, NFFT=256 with QAM Modulation Versus $\alpha$. 